Review

\title{
Current Knowledge of and Perspectives about the Pathogenesis of Blood Blister-like Aneurysms of the Internal Carotid Artery: A Review of the Literature
}

\author{
Xiao-Dong Zhai, MD1,2; Peng Hu, MD1,2; Chuan He, MD², Y Yue-Shan Feng, MD1,2; Gui-Lin Li, MD ${ }^{1,2}$; \\ Hong-Qi Zhang, MD, PhD ${ }^{1,2 \bowtie}$ \\ 1. Department of Neurosurgery, Xuanwu Hospital, Capital Medical University, Beijing, China \\ 2. China International Neuroscience Institute (China-INI), Beijing, China. \\ $\triangle$ Corresponding author: Hong-Qi Zhang, M.D., Ph.D., Department of Neurosurgery, Xuanwu Hospital, Capital Medical University, China International \\ Neuroscience Institute (China-INI), No. 45 Changchun Street, Xicheng District, Beijing, 100053. E-mail: hqzh@xwh.ccmu.edu.cn; Tel: 86+010-83198183;
} Fax:86+010-83198836.

(C) The author(s). This is an open access article distributed under the terms of the Creative Commons Attribution License (https://creativecommons.org/licenses/by/4.0/). See http://ivyspring.com/terms for full terms and conditions.

Received: 2020.09.11; Accepted: 2021.01.07; Published: 2021.03 .03

\begin{abstract}
Blood blister-like aneurysms (BBAs) are rare and usually appear at nonbranching sites in the supraclinoid portion of the internal carotid artery (ICA). Because it is difficult to obtain histological specimens of the aneurysm wall and because experimental models are challenging to establish, the pathogenesis of BBAs remains uncertain. In this paper, we reviewed the diagnostic, radiological, and pathophysiological characteristics of patients with BBAs. We also summarized the existing evidence and potential mechanisms related to the causes of BBAs. Current evidence indicates that atherosclerosis and dissection are the main prerequisites for the formation of BBAs. Hemodynamics may play a role in the process of BBA formation due to the unique vascular anatomy of the supraclinoid ICA. Further research on histopathology and hemodynamics is warranted in this field.
\end{abstract}

Key words: Blood blister-like aneurysms; Internal carotid artery; Pathogenesis; Intracranial aneurysms.

\section{Introduction}

Blood blister-like aneurysms (BBAs) usually appear at the anteromedial or anterior wall of the supraclinoid segment of the internal carotid artery (ICA) [1-4]. Although BBAs are rare, comprising approximately $0.3 \%$ to $1.0 \%$ of intracranial aneurysms (IAs), 0.5 to $2.0 \%$ of ruptured IAs and $0.9 \%$ to $6.5 \%$ of ICAs, their propensity to cause spontaneous subarachnoid hemorrhage (SAH) has led to high morbidity and mortality [5-7]. Many treatment strategies have been reported in the literature, but the management of BBAs has proven to be intractable, and the optimal therapeutic strategy for BBAs is still under debate [5-12]. The microsurgical treatments for BBAs include direct clipping, wrapping, wrap-clipping, trapping, and revascularization. Endovascular therapy can be performed with single coils, stent-assisted coils, multiple stents, and flow diversion (FD) $[5,9,10,12-14]$. During the process of separating, exposing, or clipping the BBA, the aneurysm's neck is easily torn and can cause intraoperative bleeding, leading to a poor prognosis $[13,15,16]$. The tortuous supraclinoid portion of the ICA, the fragile aneurysm wall and the small size of the aneurysm result in a lack of stability and support, which are needed for microcatheter and coiling control during endovascular treatment $[7,10,11,17]$.

BBAs typically have a thin, fragile wall and unidentifiable neck $[3,4,18]$. In contrast to saccular aneurysms, these lesions show loss of the internal elastic lamina (IEL), vascular intima and media, sometimes appearing as only a fragile fibrous layer [2, $3,6,18-20]$. Under the impact of blood flow, some BBAs can enlarge from a small protrusion to a saccular aneurysm in a short time, with a high risk of rebleeding $[1,4]$. Due to the difficulty of obtaining histological specimens of the aneurysm wall, the 
pathogenesis of BBAs remains uncertain. This work aimed to review the current literature to summarize the existing evidence and potential mechanisms related to the causes of BBAs.

\section{Diagnosis of BBAs}

In clinical practice, patients are usually diagnosed with BBAs due to the acute symptoms caused by SAH, and they typically have no complaints until rupture occurs $[6,21,22]$. BBA is frequently seen in females, younger individuals, and patients with hypertension $[5,6]$. These lesions are generally small in size and therefore do not cause symptoms of nerve compression before rupture.

From the digital subtraction angiography (DSA) images, a typical BBA is usually observed as a small irregular hemispherical protrusion on the anteromedial wall of the supraclinoid ICA. Sometimes, BBAs may be accompanied by dissection of the ICA; the lumen of the parent artery can be narrowed or dilated during angiography $[2,18,23]$. Due to their small dimensions and atypical morphological features, BBAs are often not easily detected on initial angiography [1, 24]. Therefore, three-dimensional angiography detection should be routinely performed, which is conducive to improving the detection rate of BBAs.

The diagnosis of BBAs should not be confirmed only by radiological images and clinical symptoms. Although the imaging manifestations of an aneurysm and the patient's clinical symptoms are consistent with the characteristics of BBAs, the pathological manifestations may be a true aneurysm [20, 22]. Kim et al.[20] reported a patient with SAH who had a small aneurysm at the ICA medial wall on initial angiography. Follow-up angiography 12 days later demonstrated an increased aneurysmal size and a change in morphology to a saccular shape. A histologic examination of the aneurysm wall revealed it as a true aneurysm. Zhao et al. [22] reviewed 43 small broad-necked aneurysms at the supraclinoid segment of the ICA in 41 patients who had been treated with microsurgery. The diagnosis of BBAs and non-blister aneurysms was obtained from the intraoperative inspection. Eventually, only 17 aneurysms were diagnosed as BBAs. In the strict sense, the main diagnostic criteria of BBAs should be determined by histopathological examination, with the absence of the IEL and media [4, 20, 22]. Nevertheless, it is challenging to obtain histological specimens of BBAs due to their small size and fragile wall. Therefore, the widely accepted diagnosis of BBAs is based on intraoperative inspection, during which surgeons can even observe the extremely friable aneurysmal wall.

\section{Treatment of BBAs}

Although numerous treatment strategies have been proposed, including microsurgery, endovascular treatment, and combined options, the optimal therapeutic strategy for BBAs is still under debate. Wrapping only has a limited effect on preventing postoperative re-rupture, and direct clipping carries a high risk of intraoperative rupture and subsequent ICA sacrifice [5, 12]. The direct clipping technique has not been considered a first-line treatment for the increased risk of complications [5]. Due to the small size of BBAs, the clips are unstable and may be dislocated from the original location. Wrap-clipping has the advantage of preserving the anterograde blood flow of the ICA and protecting the weakened aneurysm wall; however, they are at risk for postoperative ICA stenosis or occlusion [12, 13, 25]. Extracranial-intracranial high-flow bypass has the potential to cause ischemic complications and graft thrombosis for these patients [15, 26-28].

From the endovascular treatment perspective, the small size and unidentifiable broad-based neck of BBAs limit the effectiveness of coil embolization [5, 29, 30]. Stent-assisted coil embolization and flow diversion implantation offer low aneurysm obliteration rates in the acute phase [10, 11, 30-32]. Mokin et al. [33] reported that $42 \%(19 / 45)$ of BBAs treated by FD showed partial filling, and only $27 \%$ (12/45) showed no residual aneurysm filling at the end of the procedure. Although willis-covered stent implantation showed safe and effective clinical outcomes, ophthalmic artery (OA) or anterior choroidal artery (AchA) occlusion was observed in some patients postoperatively [34]. Overall, ruptured BBAs of the ICA are challenging to treat both microsurgically and endovascularly.

\section{Differences exist in the pathophysiological mechanisms of BBAs and saccular intracranial aneurysms (sIAs)}

Saccular intracranial aneurysms (sIAs) are pathological pouch-like dilatations of the intracranial arteries. Although BBAs can grow from a small protrusion to a saccular shape in a short period, the pathological features of BBAs are different from those of sIAs.

Under normal physiological conditions, the intracranial artery usually consists of three layers [35-38]. The inner layer is the intima with endothelial cells; the middle layer is mainly composed of smooth muscle cells (SMCs), which are embedded into a dense network of collagen and elastin fibers; and the 
adventitia mainly consists of collagen, which can provide structural integrity to the artery wall $[37,38]$.

Compared with the rarity of BBAs, sIAs are a common disease, with a prevalence of $5 \%-7 \%$ in the general population $[37,39,40]$. By establishing animal models of aneurysms or analyzing human histological specimens, the predominant model for sIAs formation and progression is obtained [35, 41, 42]. First, abnormal hemodynamic stress leads to vascular endothelial cell degeneration $[43,44]$. Inflammation is considered the leading factor in the pathogenesis of sIAs $[45,46]$. The loss of tight junction proteins between endothelial cells and the release of proinflammatory cytokines by endothelial cells leads to infiltration of a large number of inflammatory cells, causing SMCs proliferation, apoptosis, and chronic remodeling of the vascular wall [37, 45, 47-49]. The degenerated aneurysmal wall becomes too fragile to resist hemodynamic stress; as apoptosis of the IEL and SMCs and the disorganized extracellular matrix (ECM) process gradually increases, sIAs formation and growth occurs, and the aneurysm finally ruptures $[35,41,42]$.

Therefore, a true aneurysm is formed by the gradual degeneration of the artery wall, and there are

A
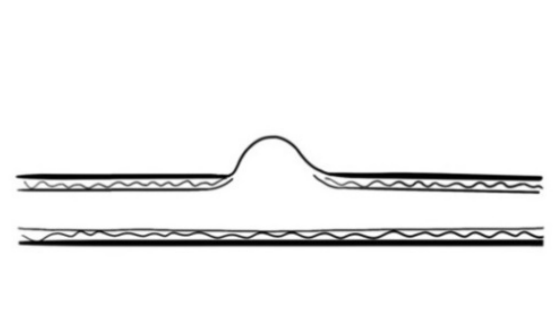

\section{Pathop}

BBAs usually show a thin, fragile wall and unidentifiable neck from the intraoperative view; these features make it challenging to obtain a specimen of the aneurysm wall, and experimental models are difficult to establish [18]. There are currently limited studies that have reported BBA's pathophysiology, and the sample size is usually small. Therefore, this substantially limits our comprehensive understanding of the mechanisms of the formation and progression of BBAs.

B
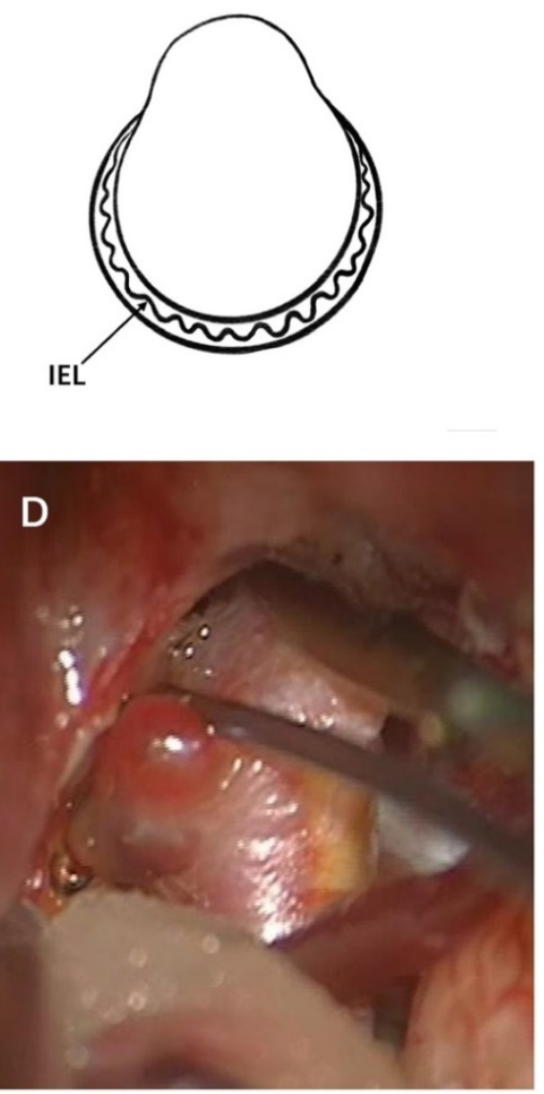

Figure 1. Illustration of a typical BBA. (A) and (B) show the internal elastic lamina, vascular intima and media have disappeared on the aneurysm wall, and the aneurysm is only covered by a fragile fibrous layer. (C) A BBA located on the right supraclinoid ICA is shown, (D) A thin and transparent aneurysm wall was observed during microsurgery. 
Dissection weakens the artery wall, making it unable to resist hemodynamic stress and eventually leading to rupture of the artery wall $[4,18]$. Ogawa et al. [1] observed significant ICA dissection in $25 \%$ $(10 / 40)$ of BBAs on angiography or during microsurgery. Sometimes, BBAs are not easily detected on initial angiography but usually show enlargement from a small hemispherical protrusion to a saccular, strawberry-shaped aneurysm in a short time. There are even studies that indicated spontaneous healing of ruptured BBAs [24, 51]. Zeineddine et al. [51] reported a case of ruptured BBA along the dorsal surface of the left ophthalmic segment. Repeat angiography two days later demonstrated receding of the ectasia, and no residual abnormality six weeks later. Due to the small size of dissection-related BBAs, focal acute arterial spasm in combination with the impact of blood flow might lead to spontaneous healing. The above evidence suggests that dissection of the artery wall is one of the fundamental prerequisites for BBAs.

Atherosclerotic remodeling, degeneration of the artery wall and loss of the IEL lead to the formation of BBAs [2, 4, 23]. Even though patients were relatively young, the cases reported by Sim et al. [23] suggested that all ten patients in their study had atherosclerotic parent arteries adjacent to the BBAs. Ishikawa et al. [2] reported that the ICA wall's arteriosclerosis was observed from an autopsy, and the IEL and media disappeared between the normal and sclerotic ICA walls. These pathophysiological findings, suggest that atherosclerosis causes degeneration of the IEL in the arteries and eventually leads to rupture of the arterial wall. Ulceration associated with atherosclerotic plaques can lead to the destruction of the IEL in the vessel wall and allow hematoma formation within the media of the artery wall, leading to the formation of BBAs.

There exist only a few reports of traumatic-related BBAs. Haji et al. [52] reported one case of cranial trauma with diffuse basal SAH. The computerized tomography angiography (CTA) demonstrated a small, 2-mm BBA from the left supraclinoid ICA's dorsal surface at a nonbranching site. The pathology of the native ICA was observed during microsurgery, raising the possibility of a preexisting BBA. Segmental arterial mediolysis (SAM) is a rare nonatherosclerotic, noninflammatory vascular disease [53]. Pickup et al. [54] suggested SAM to be a condition found in Ehlers-Danlos type IV. A small number of SAM-related BBA cases have been reported, which manifest as lysis of the tunica media, smooth muscle degeneration and serration of the lamina elastica interna $[55,56]$.

\section{Hemodynamic characteristics}

sIAs commonly arise at the bifurcations of the cerebral arteries. In contrast, BBAs usually appear at nonbranching sites in the surpraclinoid portion of the ICA $[1,18,22]$. A number of scholars have argued that hemodynamics may play an important role in the formation of BBAs. The hemodynamic characteristics caused by the unique vascular anatomy of the carotid siphon and the formation of BBAs may have some degree of correlation. To our knowledge, the current studies were not carried out with sufficient evidence from hemodynamic studies to draw corresponding conclusions on the mechanisms of BBA formation.

The large curvature of the carotid siphon is one of its essential characteristics. In a computational fluid dynamics (CFD) simulation study, high curvature tightness resulted in a significantly higher wall shear stress (WSS) on the outer wall than on the inner wall of the bend [57]. Besides, Meng et al.[43] analyzed the morphological and hemodynamics characteristics of aneurysms, suggesting that a high WSS is significantly correlated with a small thin-walled and entirely translucent aneurysm. Despite similar appearances, further hemodynamic studies that provide more direct evidence are required to clarify this presumption.

The OA is one of the main branches of the paraclinoid ICA. The branching vessels can affect the hemodynamic characteristics of the ICA trunk through shunting. Indo et al. [58] indicated that ICA anterior wall aneurysms in patients with an OA anomalous origin tend to be saccular aneurysms with normal neck walls. On the other hand, of the ten patients who underwent microsurgery, all six aneurysms with a normal OA showed dissecting aneurysms or BBAs, not saccular aneurysms. It is necessary to conduct further hemodynamic analyses in the future to help explore the pathogenesis of BBAs.

\section{Conclusion}

In this study, we reviewed the diagnostic, radiological, and pathophysiological characteristics of patients with BBAs. We also summarized the existing evidence and potential mechanisms related to the causes of BBAs of the supraclinoid ICA. Current evidence indicates that atherosclerosis and dissection are the main prerequisites for the formation of BBAs. Hemodynamics may play a role in the process of BBA formation due to the unique vascular anatomy of the supraclinoid ICA. Overall, the pathogenesis of BBAs is not entirely clear and further research on their histopathology and hemodynamics is warranted. 


\section{Abbreviations}

BBAs: blood blister-like aneurysms; ICA: internal carotid artery; IAs: intracranial aneurysms; SAH: subarachnoid hemorrhage; IEL: internal elastic lamina; DSA: digital subtraction angiography; CTA: computerized tomography angiography; OA: ophthalmic artery; AchA: anterior choroidal artery; SMCs: smooth muscle cells; sIAs: saccular intracranial aneurysms; ECM: extracellular matrix; SAM: segmental arterial mediolysis; CFD: computational fluid dynamics; WSS: wall shear stress.

\section{Acknowledgements}

\section{Funding}

This work was supported by the National Key R\&D program of China with grant 2016YFC1300800 and the Beijing Municipal Administration of Hospitals' Ascent Plan with grant DFL20180801.

\section{Competing Interests}

The authors have declared that no competing interest exists.

\section{References}

1. Ogawa A, Suzuki M and Ogasawara K. Aneurysms at nonbranching sites in the surpaclinoid portion of the internal carotid artery: internal carotid artery trunk aneurysms. Neurosurgery. 2000; 47: 578-583.

2. Ishikawa T, Nakamura N, Houkin $\mathrm{K}$, et al. Pathological consideration of a "blister-like" aneurysm at the superior wall of the internal carotid artery: Case report. Neurosurgery. 1997; 40: 403-405.

3. Nakagawa F, Kobayashi S, Takemae T, et al. Aneurysms protruding from the dorsal wall of the internal carotid artery. J Neurosurg. 1986; 65: 303-308.

4. Abe M, Tabuchi K, Yokoyama $\mathrm{H}$, et al. Blood blisterlike aneurysms of the internal carotid artery. J Neurosurg. 1998; 89: 419-424.

5. Peschillo S, Cannizzaro D, Caporlingua A, et al. A Systematic Review and Meta-Analysis of Treatment and Outcome of Blister-Like Aneurysms. AJNR Am J Neuroradiol. 2016; 37: 856-861.

6. Regelsberger J, Matschke J, Grzyska U, et al. Blister-like aneurysms--a diagnostic and therapeutic challenge. Neurosurg Rev. 2011; 34: 409-416.

7. Hao X, Li G, Ren J, et al. Endovascular Patch Embolization for Blood Blister-Like Aneurysms in Dorsal Segment of Internal Carotid Artery. World Neurosurg. 2018; 113: 26-32.

8. McLaughlin N, Laroche M and Bojanowski MW. Blister-like aneurysms of the internal carotid artery - management considerations. Neurochirurgie. 2012; 58: 170-186

9. Zhu D, Yan Y, Zhao P, et al. Safety and Efficacy of Flow Diverter Treatment for Blood Blister-Like Aneurysm: A Systematic Review and Meta-Analysis. World Neurosurg. 2018; 118: e79-e86.

10. Zhu D, Fang Y, Yang P, et al. Overlapped Stenting Combined with Coiling for Blood Blister-Like Aneurysms: Comparison of Low-Profile Visualized Intraluminal Support (LVIS) Stent and Non-LVIS Stent. World Neurosurg. 2017; 104: 729-735.

11. Fang Y, Zhu D, Peng Y, et al. Treatment of Blood Blister-like Aneurysms with Stent-Assisted Coiling: A Retrospective Multicenter Study. World Neurosurg. 2019; 126: e486-e491.

12. Meling TR. What are the treatment options for blister-like aneurysms? Neurosurg Rev. 2017; 40: 587-593.

13. Meling TR, Patet G. Clip-wrapping of ruptured blood blister-like aneurysms of the internal carotid artery. Neurosurg Rev. 2020;43(5):1365-71.

14. Pahl FH, de Oliveira MF, Teles Gomes Mde Q, et al. Blister-Like Aneurysms: Report of Successful Surgical Treatment of Consecutive Cases and Review of the Literature. World Neurosurg. 2016; 89: 376-381.

15. Sorimachi T, Osada T, Hirayama A, et al. Preservation of Anterior Choroidal Artery Blood Flow During Trapping of the Internal Carotid Artery for a Ruptured Blood Blister-Like Aneurysm with High-Flow Bypass. World Neurosurg. 2019; 122: e847-e855.

16. Nishi T, Kaji M, Koga K, et al. Clipping on Crossed Wrapping Method for Ruptured Blood Blister-Like Aneurysm of the Internal Carotid Artery: Technical Note and Long-Term Results. World Neurosurg X. 2019; 2: 100005.
17. Wu YQ, Li LZ, Wang ZY, et al. Endovascular Intervention with a Low-profile Visualized Intraluminal Support Stent Versus Surgical Clipping for Blood Blister-like Aneurysms: A Retrospective Study. Clin Neuroradiol. 2020.

18. Lee SU, Kwak Y, Oh CW, et al. Pathogenesis of dorsal internal carotid artery wall aneurysms based on histopathologic examination and microscopic configuration. J Clin Neurosci. 2018; 58: 181-186.

19. Sundt TM, Jr. and Murphey F. Clip-grafts for aneurysm and small vessel surgery. 3. Clinical experience in intracranial internal carotid artery aneurysms. J Neurosurg. 1969; 31: 59-71.

20. Kim JH, Kwon $\mathrm{TH}$, Kim JH, et al. Internal carotid artery dorsal wall aneurysm with configurational change: Are they all false aneurysms? Surg Neurol. 2006; 66: 441-443; discussion 443

21. Szmuda T, Sloniewski P, Waszak PM, et al. Towards a new treatment paradigm for ruptured blood blister-like aneurysms of the internal carotid artery? A rapid systematic review. J Neurointerv Surg. 2016; 8: 488-494.

22. Zhao Y, Zhang Q, Wang S, et al. Comparison of radiological and clinical characteristics between blood blister-like aneurysms (BBAs) and non-blister aneurysms at the supraclinoid segment of internal carotid artery. Neurosurg Rev. 2019; 42: 549-557.

23. Sim SY, Shin YS, Cho KG, et al. Blood blister-like aneurysms at nonbranching sites of the internal carotid artery. J Neurosurg. 2006; 105: 400-405.

24. Ueta $\mathrm{T}$, Ichi $\mathrm{S}$, Ochi $\mathrm{T}$, et al. Spontaneous regression of an aneurysm at a nonbranching site of the supraclinoid internal carotid artery. Case report. J Neurosurg. 2004; 101: 1070-1072.

25. Hanihara M, Yoshioka H, Kanemaru $\mathrm{K}$, et al. Long-Term Clinical and Angiographic Outcomes of Wrap-Clipping for Ruptured Blood Blister-Like Aneurysms of the Internal Carotid Artery Using Advanced Monitoring. World Neurosurg. 2019; 126: e439-e446.

26. Kawashima A, Okada Y, Kawamata T, et al. Successful treatment of a blood blister-like aneurysm of the internal carotid artery by trapping with a high-flow bypass. J Clin Neurosci. 2008; 15: 797-800.

27. Kazumata K, Nakayama N, Nakamura T, et al. Changing treatment strategy from clipping to radial artery graft bypass and parent artery sacrifice in patients with ruptured blister-like internal carotid artery aneurysms. Neurosurgery. 2014; 10(Suppl 1): 66-72; discussion 73.

28. Kikkawa Y, Ikeda T, Takeda R, et al. Results of Early High-Flow Bypass and Trapping for Ruptured Blood Blister-Like Aneurysms of the Internal Carotid Artery. World Neurosurg. 2017; 105: 470-477.

29. Chen R, Xiao A, Li H, et al. Blood blister-like aneurysms in Tibetans: A retrospective observational study. Clin Neurol Neurosurg. 2017; 156: 18-23.

30. Park JH, Park IS, Han DH, et al. Endovascular treatment of blood blister-like aneurysms of the internal carotid artery. J Neurosurg. 2007; 106: 812-819.

31. Rouchaud A, Brinjikji W, Cloft HJ, et al. Endovascular Treatment of Ruptured Blister-Like Aneurysms: A Systematic Review and Meta-Analysis with Focus on Deconstructive versus Reconstructive and Flow-Diverter Treatments. AJNR Am J Neuroradiol. 2015; 36: 2331-2339.

32. Fang C, Tan HQ, Han HJ, et al. Endovascular isolation of intracranial blood blister-like aneurysms with Willis covered stent. J Neurointerv Surg. 2017; 9: 963-968.

33. Mokin M, Chinea A, Primiani CT, et al. Treatment of blood blister aneurysms of the internal carotid artery with flow diversion. J Neurointerv Surg. 2018; 10: 1074-1078.

34. Liu Y, Yang HF, Xiong ZY, et al. Efficacy and Safety of Willis Covered Stent for Treatment of Complex Vascular Diseases of the Internal Carotid Artery. Ann Vasc Surg. 2019; 61: 203-211.

35. Marbacher S, Marjamaa J, Bradacova K, et al. Loss of mural cells leads to wall degeneration, aneurysm growth, and eventual rupture in a rat aneurysm model. Stroke. 2014; 45: 248-254.

36. Frosen J. Smooth muscle cells and the formation, degeneration, and rupture of saccular intracranial aneurysm wall--a review of current pathophysiological knowledge. Transl Stroke Res. 2014; 5: 347-356.

37. Chalouhi N, Hoh BL and Hasan D. Review of cerebral aneurysm formation, growth, and rupture. Stroke. 2013; 44: 3613-3622.

38. Etminan N, Buchholz BA, Dreier R, et al. Cerebral aneurysms: formation, progression, and developmental chronology. Transl Stroke Res. 2014; 5: $167-173$

39. Korja M, Kivisaari R, Rezai Jahromi B, et al. Size and location of ruptured intracranial aneurysms: consecutive series of 1993 hospital-admitted patients. J Neurosurg. 2017; 127: 748-753.

40. Li MH, Chen SW, Li YD, et al. Prevalence of unruptured cerebral aneurysms in Chinese adults aged 35 to 75 years: a cross-sectional study. Ann Intern Med. 2013; 159: 514-521.

41. Kataoka H. Molecular mechanisms of the formation and progression of intracranial aneurysms. Neurol Med Chir (Tokyo). 2015; 55: 214-229.

42. Chalouhi N, Ali MS, Jabbour PM, et al. Biology of intracranial aneurysms: role of inflammation. Journal of Cerebral Blood Flow and Metabolism. 2012. 32. $1659-1676$

43. Meng H, Tutino VM, Xiang J, et al. High WSS or low WSS? Complex interactions of hemodynamics with intracranial aneurysm initiation, growth, and rupture: toward a unifying hypothesis. AJNR Am J Neuroradiol. 2014; 35: 1254-1262.

44. Can A and Du R. Association of Hemodynamic Factors with Intracranial Aneurysm Formation and Rupture: Systematic Review and Meta-analysis. Neurosurgery. 2016; 78: 510-520. 
45. Hosaka K and Hoh BL. Inflammation and cerebral aneurysms. Transl Stroke Res. 2014; 5: 190-198. 2013/12/11. DOI: 10.1007/s12975-013-0313-y.

46. Tulamo R, Frosen J, Hernesniemi J, et al. Inflammatory changes in the aneurysm wall: a review. J Neurointerv Surg. 2010; 2: 120-130.

47. Jamous MA, Nagahiro S, Kitazato KT, et al. Endothelial injury and inflammatory response induced by hemodynamic changes preceding intracranial aneurysm formation: experimental study in rats. J Neurosurg. 2007; 107: 405-411.

48. Fan $\mathrm{XJ}, \mathrm{Zhao} \mathrm{HD}, \mathrm{Yu}$ G, et al. Role of inflammatory responses in the pathogenesis of human cerebral aneurysm. Genet Mol Res 2015; 14: 9062-9070.

49. Tada Y, Yagi K, Kitazato KT, et al. Reduction of endothelial tight junction proteins is related to cerebral aneurysm formation in rats. Journal of Hypertension. 2010; 28: 1883-1891.

50. Tanoue S, Kiyosue H, Matsumoto S, et al. Ruptured "blisterlike" aneurysm with a pseudoaneurysm formation requiring delayed intervention with endovascular coil embolization. Case report. J Neurosurg. 2004; 101: 159-162.

51. Zeineddine HA, Jones $\mathrm{W}$, Conner $\mathrm{CR}$, et al. Spontaneous Healing of a Ruptured Blood Blister-Like Aneurysm. World Neurosurg. 2018; 119: 85-88.

52. Haji FA, Boulton MR and de Ribaupierre S. Blister-like supraclinoid internal carotid artery pseudoaneurysm in a 15-year-old male: case report and review of the literature. Pediatric neurosurgery. 2011; 47: 449-454.

53. Slavin RE and Gonzalez-Vitale JC. Segmental mediolytic arteritis: a clinical pathologic study. Laboratory investigation; a journal of technical methods and pathology. 1976; 35: 23-29.

54. Pickup MJ and Pollanen MS. Traumatic subarachnoid hemorrhage and the COL3A1 gene: emergence of a potential causal link. Forensic science, medicine, and pathology. 2011; 7: 192-197.

55. Hellstern V, Aguilar Perez M, Kohlhof-Meinecke $\mathrm{P}$, et al. Concomitant Retroperitoneal and Subarachnoid Hemorrhage Due to Segmental Arterial Mediolysis: Case Report and Review of the Literature. Clin Neuroradiol. 2018; 28: $445-450$.

56. Tanaka K, Fujiwara M, Okuda Y, et al. A Ruptured Blood Blister-Like Aneurysm Associated with Intraperitoneal Hemorrhage Due to Segmental Arterial Mediolysis: A Case Report And Literature Review. World Neurosurg. 2019; 134: 79-85.

57. Lauric A, Hippelheuser J, Safain MG, et al. Curvature effect on hemodynamic conditions at the inner bend of the carotid siphon and its relation to aneurysm formation. Journal of biomechanics. 2014; 47: 3018-3027.

58. Indo $\mathrm{M}$, Oya $\mathrm{S}$, Tanaka $\mathrm{M}$, et al. High incidence of ICA anterior wall aneurysms in patients with an anomalous origin of the ophthalmic artery: possible relevance to the pathogenesis of aneurysm formation. J Neurosurg. 2014; 120: 93-98. 